\title{
Phase behavior of the hard-sphere Maier-Saupe fluid under spatial confinement
}

\author{
N. G. Almarza, C. Martín, and E. Lomba \\ Instituto de Química Física Rocasolano, CSIC, Serrano 119, E-28006 Madrid, Spain
}

(Received 2 June 2009; published 3 September 2009)

\begin{abstract}
The Maier-Saupe hard-sphere fluid is one of the simplest models that accounts for the isotropic-nematic transition characteristic of liquid crystal phases. At low temperatures the model is known to present a gasliquid-like transition with a large difference between the densities of the coexistence phases, whereas at higher temperature the transition becomes a weak first-order transition resembling the typical order-disorder (nematicisotropic) phase change of liquid crystals. Spatial dimensionality directly conditions the character of the orientational phase change (i.e., the high temperature transition), that goes from a first-order transition in the purely three-dimensional case, to a Berezinskii-Kosterlitz-Thouless-like continuous transition which occurs when the three dimensional Maier-Saupe spins are constrained to lie on a plane. In the latter instance, the ordered phase is not endowed with true long-range order. In this work we investigate how the continuous transition transforms into a true first-order phase change, by analyzing the phase behavior of a system of three dimensional Maier-Saupe hard spheres confined between two parallel plates, with separations ranging from the quasi-two-dimensional regime to the bulk three-dimensional limit. Our results indicate that spatial confinement in one direction induces the change from first order to a continuous transition with a corresponding decrease of the transition temperatures. As to the gas-liquid transition, the estimated "critical" temperatures and densities also decrease as the fluid is confined, in agreement with previous results for other simple systems.
\end{abstract}

DOI: 10.1103/PhysRevE.80.031501

PACS number(s): 64.70.-p, 64.60.Cn, 61.20.Gy

\section{INTRODUCTION}

In a series of recent papers [1-3], the authors in collaboration with Lado, have revisited the phase behavior of one of the simplest continuum interaction models that reproduces the isotropic-nematic (IN) transition so characteristic of liquid crystal materials, namely, the Maier-Saupe (MS) hardsphere fluid $[4,5]$. The three-dimensional version of this model has been known since long to undergo a weak firstorder transition, either in a lattice- known as LebwohlLasher model [6-9]—or in the continuum [10]. In contrast, when the Maier-Saupe spins are spatially constrained to lie on a plane-even if the spins themselves are free to rotate out of the plane- - the first-order transition switches to a continuum order-disorder transition, in which the ordered phase lacks a complete long range order, at least if no density fluctuations are allowed (Lebwohl-Lasher model with full occupancy of the lattice sites) [11-13] or if the temperature is high [1-3]. Although the precise nature of the transition is still under some controversy [13], it seems that the process of formation of the quasi-long-range ordered (QLRO) phase, is accompanied by changes in heat capacities and orderparameter susceptibilities that bear some resemblance with the situation found in the Berezinskii-Kosterlitz-Thouless (BKT) transition $[14,15]$. This was confirmed for continuum models in Ref. [1] also in the presence of a disorienting field, and further on in Ref. [3] for a family of planar [twodimensional (2D)] systems in which the dimensionality of the orientational degrees of freedom of the Maier-Saupe spins was varied from two to four. These models are known as $R P^{n-1}$ (real projective space in $n$ dimensions) where $n$ $=2,3$, and 4 . The $R P^{2}$-model considered in [3] is essentially the same one treated in [1] in the presence of an external field and is just the continuum version of the models dealt with in Refs. [11-13].
Summarizing the results for continuum systems, the fluid phase equilibria of the three-dimensional MS system was studied in detail in [2] by means of Monte Carlo (MC) simulation. As mentioned, in the fluid phase one finds a certain range of temperatures in which an I-N phase transition appears. Outside that range, both at low and high temperatures, the compression of the isotropic phase will induce a transition to either a crystalline solid (S) phase or a plastic solid (PS) (without crossing a region of nematic stability). When temperatures are sufficiently low, the difference in density between the isotropic and nematic phases is large enough, so that the transition might also be thought of as a gas-liquid phase change induced by the average net attraction of the MS interaction [3]. The condensed phase exhibits nematic order. In agreement with previous works [6-10], in three dimensions the I-N transition is found [2] to be first order, becoming weaker as the temperature increases. The I-N transition exists as a thermodynamic stable transition up to a high temperature triple point where the I-N, N-S, and I-S transition lines meet. The solid phase is also expected to undergo a weak first-order transition between PS and S phases.

The continuum two dimensional MS (or $R P^{2}$ ) case has also been studied in depth using MC simulations [1]. This system can be considered as the limiting case of a threedimensional nematogenic system confined between two parallel walls. The phase diagram is partially similar to that of three-dimensional (3D) systems. At low temperature (but not too low) a first-order transition occurs between a low density isotropic phase and a high density fluid phase. This high density phase exhibits nematic order at the scale of the system sizes used in the simulation, but it is supposed to have quasi-long-range-order $[9,16]$ in the thermodynamic limit, and in fact one observes that the magnitude of the order parameters decreases as the sample size increases [1]. As the temperature increases the density of the two phases approach 
until they meet at an apparent multicritical point. For higher temperatures the transition is thought to be of BKT type [1].

It is obvious then that spatial confinement substantially changes the nature of the phase transition. It is known that quantities such as critical temperatures and densities will be strongly affected by confinement [17], by the simple fact that the average number of neighbors near the system boundaries will be different from that of the bulk. So in the case of fluids confined in purely repulsive slit pores (which is the case we will be dealing with) vapor-liquid critical temperatures and densities will decrease as the interwall separation shrinks. Also, at least in simple fluids, when going from a bulk 3D fluid to a system confined in a planar surface (2D), the shape of the vapor-liquid curve changes when switching from 3D to $2 \mathrm{D}$ Ising universality class. The effects of spatial confinement of nematogens were already subject of investigation by Cleaver and Allen [18], which showed that the transition temperature from isotropic to nematic phase, $T_{I N}$ decreased with confinement for a Lebwohl-Lasher lattice model of spins in a slab geometry. This model was further studied by Telo da Gama et al. [19,20] using mean field and a Bethetype approximation. Telo da Gama and Tarazona [20] proposed a mechanism for the crossover between first-order and continuous transitions based on the dimensional crossover of both space and order-parameter variables, carrying out explicit mean field calculations.

The aim of this paper is to offer a detailed analysis of the switching from a first order to a continuous transition induced by confinement in the continuum hard-sphere MS fluid. To that purpose we will present a full account of the evolution of order parameters, their corresponding susceptibilities, heat capacities, and in some cases critical exponents, using extensive MC simulations and efficient sampling and analysis tools, such as cluster moves and histogram reweighting. Calculations will be carried out using various interwall separations and the results analyzed in the context of the bulk system behavior. In this work we will restrict our study to inert walls that induce no alignment on their neighboring spins. We will see in turn how spatial confinement affects the vapor-liquid (or dilute fluid-dense fluid) equilibrium, following the regular trends already encountered for fluids in slit pores with repulsive walls [17].

The rest of the paper is sketched as follows. In the next section we introduce the model and simulation conditions. Details of the methods to evaluate the first-order transitions are presented in Sec. III together with the analysis of our calculations. In Sec. IV we comment on the procedures utilized to analyze the order-disorder transition in the continuous regime and the approach followed to locate the apparent multicritical points, where the low temperature first-order transition meets the continuous high temperature isotropicnematic transition line. The results of these calculations are also discussed in this section. Finally, the complete phase diagram for the confined systems is presented in Sec. V and discussed in connection with the results for the bulk threedimensional MS fluid and the corresponding MS system confined to a plane (continuous $\mathrm{R} P^{2}$ model).

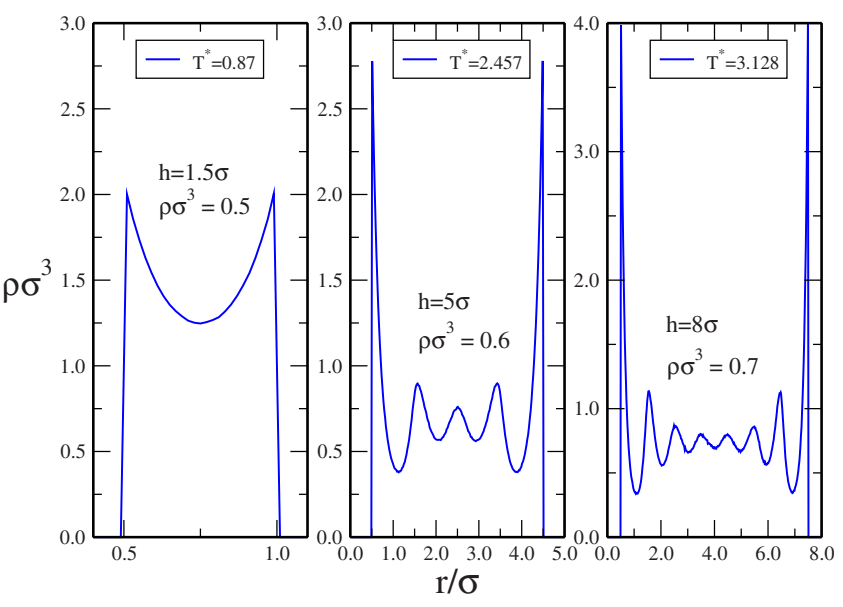

FIG. 1. (Color online) Density profile of a MS hard-sphere fluid confined in a slit pore with varying pore widths.

\section{MODEL AND SIMULATION PROCEDURES}

Our system of interest is defined by a set of hard-sphere particles with embedded Maier-Saupe spins, whose total interaction energy is given by

$$
U=\sum_{i<j} u_{H S}\left(r_{i j}\right)+\sum_{i<j} u\left(r_{i j}, \mathbf{s}_{i}, \mathbf{s}_{j}\right),
$$

where $u_{H S}\left(r_{i j}\right)$ is a hard core repulsion between spheres $i$ and $j$ of diameter $\sigma, \mathbf{s}_{i}$ is a 3D unit vector that denotes the orientation of the spin $i$, and the Maier-Saupe interaction is given by

$$
u\left(r, \omega_{i}, \omega_{j}\right)=-K u_{0}(r) P_{2}\left(\cos \theta_{i j}\right)
$$

with $\cos \theta_{i j}=\mathbf{s}_{i} \cdot \mathbf{s}_{j}$ and

$$
\begin{aligned}
u_{0}(r)= & \frac{\exp [-\kappa(r / \sigma)-1]}{r / \sigma} \\
& -\frac{\exp \left[-\kappa\left(R_{c} / \sigma\right)-1\right]}{R_{c} / \sigma} \text { if } \sigma<r<R_{c}
\end{aligned}
$$

and $u_{0}=0$ otherwise. We have truncated the interaction at $R_{c}=4 \sigma$ for computational convenience. $P_{2}(x)=\left(3 x^{2}-1\right) / 2$ is the second degree Legendre polynomial and the coupling constant $K$ is used here to define the energy units, so that the reduced temperature is given by $T^{*}=k_{B} T / K$, with $k_{B}$ being Boltzmann's constant. The screening parameter has been set to unity, $\kappa=1$. As shown in Ref. [13], the appropriate Mayer averaging of this potential leads to an effective attractive interaction, by which one might expect at sufficiently low temperatures to encounter a vapor-liquid transition, even without the explicit presence of dispersive interactions. Now, the phase behavior of the system as defined above, was fully studied in the bulk in Ref. [2], using MC simulation and an inhomogeneous integral equation approach. In this paper, our system particles will be confined between two parallel repulsive walls, separated a distance $h$ (with $h=1.5 \sigma, 5 \sigma$, and $8 \sigma$ ). In Fig. 1 we can see the density profiles for these interwall separations and various densities, $\rho \sigma^{3}$.

As found in previous works, various simulation techniques are needed depending on the part of the phase dia- 
gram one is interested on. Calculations for the vapor-liquid equilibrium mostly rest on the use of canonical (NVT) Monte Carlo simulation. To that purpose a Markov chain is generated using $N$ translational attempts per cycle (being $N$ the number of particles), accepted or rejected following the standard Metropolis algorithm. Then orientational moves are performed using a combination of a local update algorithm $[1,21]$ and cluster moves $[1,22]$, in order to minimize critical slowdown effects when approaching the vicinity of critical points. In order to study the isotropic-nematic transition at higher temperatures (which is known to be weakly first order [2]), we resorted to constant pressure $(N p T)$ simulations, in which together with orientational and translational moves, volume changes are incorporated in a standard way [23]. Now, at low interwall separations, the high temperature isotropic-nematic transition (which will be shown to be continuouslike in this case) merges with the low temperature first-order vapor-liquid transition at an apparent multicritical point. The precise location of these apparent multicritical points is undertaken using a more sophisticated approach based on the Grand-Canonical Wang-Landau's methodology $[1,24,25]$, which will be presented in further detail in Sec. IV A.

The choice of techniques herein employed is inspired by those extensively and successfully used to study 2D and 3D Maier-Saupe spins both in lattice $[11,12]$ and in continuum models [1-3]. Obviously, when focusing on continuum systems, limitations of sample size will be more restrictive that in the case of lattice models. According to our previous experience, the sample sizes used in our calculations are sufficiently large to yield reasonably reliable estimates when applying finite size scaling procedures.

\section{ANALYSIS OF FIRST-ORDER TRANSITIONS}

As mentioned before, the Maier-Saupe interaction term, induces on the average an attractive term in the effective interaction between two spins. This might give rise to a vapor-liquid first-order transition, in which the density difference between the two phases is substantial. On the other hand, at high temperatures, at least in the three dimensional bulk, the isotropic-nematic transition is always first order in the absence of external fields [20]. Both the vapor-liquid and the isotropic-nematic transitions are coupled in a continuous fashion [2], but the methods to deal with each region of the phase diagram are different. In the case of the confined system, we will see that at sufficiently high temperatures the isotropic-nematic transition can be considered continuous, but for temperatures in the immediate neighborhood below the apparent multicritical point (where the first order and the continuous transition meet), one has to use the same approaches as in the high temperature bulk system, given the small density difference between the ordered and disordered phases.

\section{A. Vapor-liquid transition}

The calculation of the vapor-liquid (or low density isotropic-high density nematic phase) coexistence curves was carried out by means of extensive $N V T$ simulations with various sample sizes $(N=256,500,864$ and 1372 particles for pore sizes $h<8 \sigma$, and 500, 864 and 1372 particles for $h=8 \sigma$ ). The pressure along the $y$ or $z$ direction (the system is confined along the $x$-direction), is given by

$$
\beta p_{y z}=\rho+\beta p_{y z}^{H S}+\beta p_{y z}^{a t t},
$$

where $\beta=1 / k_{B} T$, with $T$ being the temperature and $k_{B}$, Boltzmann's constant, and $\rho$ is the number density (i.e., the ideal gas contribution). The attractive contribution to the virial along the $y$ or $z$ direction, $p_{y z}^{a t t}$, can be evaluated in a straightforward fashion [23]. As to the hard core term, $\beta p_{y z}^{H S}$, it can also be readily evaluated by means of the volume perturbation approach exploited in [26,27]. According to this strategy, the volume of the simulation cell is perturbed, shrinking its sides of length, $l_{y z}$, to a new length $l_{y z}^{\prime}=l_{y z}(1-\Delta)$-in our case $\Delta=5 \times 10^{-4}$, and $l_{y}=l_{z}$-and the perturbed volume is $V_{\text {per }}=h l_{y}^{\prime} l_{z}^{\prime}$, being the original unperturbed volume, $V=h l_{y} l_{z}$. Counting the number of overlaps induced by the perturbation, $N_{o v}$, the hard core contribution to the pressure is given by

$$
\beta p_{y z}^{H S}=\left\langle\frac{N_{o v}}{V-V_{p e r}}\right\rangle,
$$

where $\langle\cdots\rangle$ denotes the ensemble average. Using calculations for a relatively wide range of densities (from $\rho \sigma^{3}$ $=0.05$ to 0.85 with a grid $\delta \rho \sigma^{3}=0.05$ ), one proceeds to perform a weighted least-squares fit of the pressures to a virial equation of the form

$$
\beta p(\rho)=\rho+\sum_{k=0}^{k_{\max }} B_{k+2} \rho^{k+2},
$$

which can be integrated to yield the chemical potential

$$
\beta \mu(\rho)=\ln \rho+\sum_{k=0}^{k_{\max }} \frac{k+2}{k+1} B_{k+2} \rho^{k+2} .
$$

Using a $k_{\max }$ such that minimizes the value of the $\chi^{2}$ per degree of freedom [28], we have determined vapor-liquid coexistence curves solving the equilibrium equations

$$
\begin{aligned}
& \beta p\left(\rho_{v}, T ; N\right)=\beta p\left(\rho_{l}, T ; N\right), \\
& \beta \mu\left(\rho_{v}, T ; N\right)=\beta \mu\left(\rho_{l}, T ; N\right) .
\end{aligned}
$$

The values so obtained for a series of system sizes are then extrapolated to $N \rightarrow \infty$. As temperature increases, the density of the vapor phase raises, and one can bypass the thermodynamic integration from very low densities performing an integration of the pressure from a reference value $\rho_{0}$ (such that $\rho_{0}$ is smaller than the expected $\rho_{v}$ ), and fitting $\beta p$ to a polynomial of $\rho$, leading to an expression for $\beta \mu$ different from Eq. (6). The use of a reference $\rho_{0}$ just introduces constant terms of both sides of Eq. (8) that cancel out.

\section{B. High temperature isotropic-nematic transition}

For interwall separations $h>1.5 \sigma$ and relatively high temperatures, the coexistence densities are too close and the 


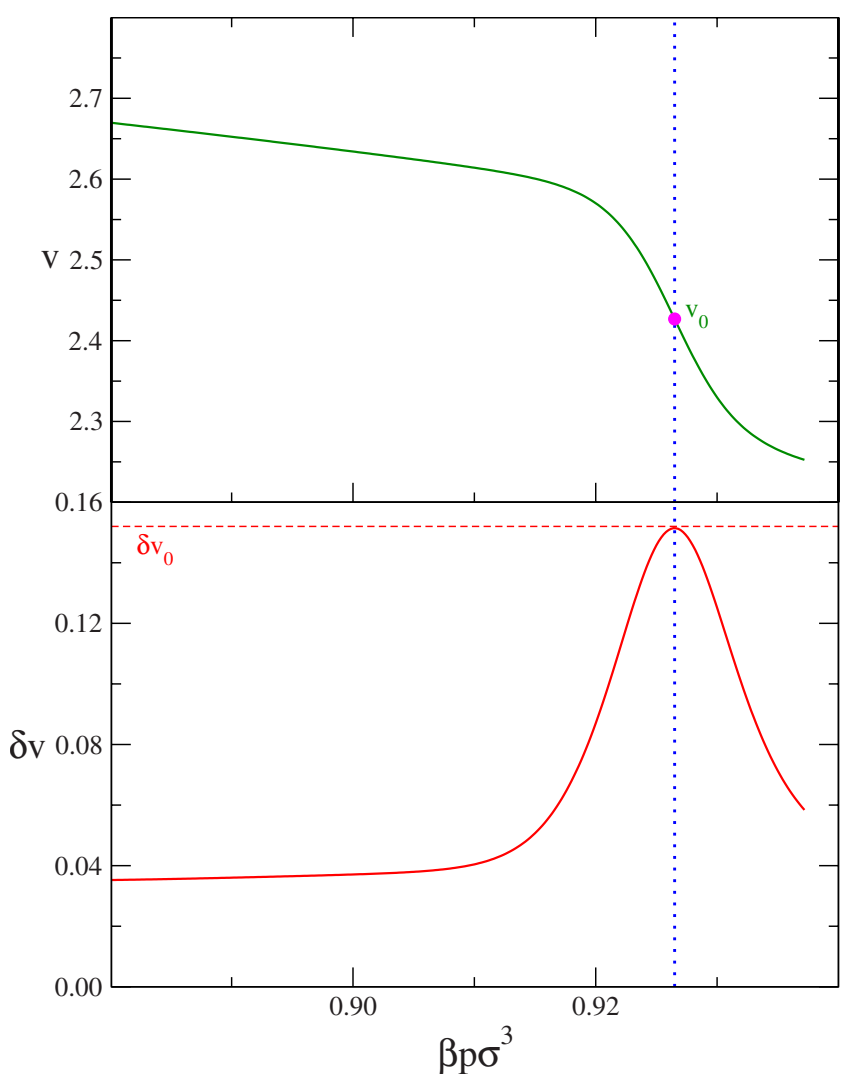

FIG. 2. (Color online) Graphical determination of the vaporliquid coexistence densities in an $N p T$ simulation (see Eq. (10).

NVT thermodynamic integration approach sketched above fails. Thus, for $h=5 \sigma$ and $T^{*} \geq 1.55$, and for $h=8 \sigma$ and $T^{*}$ $\geq 1.80$ we have resorted to the use of simulations in the $N p T$ ensemble, using 256, 500, 864, and 1372 particles and several values of $\beta p$ in the neighborhood of the expected transition. Using histogram reweighting techniques $[2,29,30]$ the results for various $\beta p$ are combined to locate the transition. The determination of the vapor-liquid coexistence is based on the analysis of the behavior of the volume per particle, $v$, and its fluctuations [2]

$$
\delta v=\left\langle\left(v-v_{0}\right)^{2}\right\rangle^{1 / 2} \quad \text { with } v_{0}=\langle v\rangle,
$$

which for a given $\beta p_{0}$ reaches a maximum (see Fig. 2), $\delta v_{0}$. Then, the coexistence densities will be given by

$$
\rho_{v}=\frac{1}{v_{0}+\delta v_{0}} \quad \text { and } \rho_{l}=\frac{1}{v_{0}-\delta v_{0}} .
$$

As in the case of the thermodynamic integration approach, results from various sample sizes are extrapolated to the thermodynamic limit. Additionally, the behavior of the cumulant

$$
G_{4}=\frac{\left\langle\left(v-v_{0}\right)^{4}\right\rangle}{\left\langle\left(v-v_{0}\right)^{2}\right\rangle^{2}}
$$

provides an assessment as to the order of the transition. Thus, if $G_{4} \rightarrow 1$ as $N \rightarrow \infty$ the transition is first order, whereas at the critical point its value depends on the universality class of the system [31]. For temperatures above the critical, $G_{4} \simeq 3$.
TABLE I. First-order transition coexistence densities and pressures for the confined MS fluid for interwall separation $h=1.5 \sigma$. TI stands for thermodynamic integration from $N V T$ simulations

\begin{tabular}{ccccc}
\hline \hline$T^{*}$ & $\beta p \sigma^{3}$ & $\rho_{v} \sigma^{3}$ & $\rho_{l} \sigma^{3}$ & Method \\
\hline 0.400 & $0.066(5)$ & $0.060(4)$ & $0.598(3)$ & TI \\
0.450 & $0.126(3)$ & $0.112(4)$ & $0.558(3)$ & TI \\
0.500 & $0.224(6)$ & $0.186(3)$ & $0.508(6)$ & TI \\
0.525 & $0.280(1)$ & $0.229(3)$ & $0.480(8)$ & TI \\
0.550 & $0.336(7)$ & $0.268(10)$ & $0.444(15)$ & TI \\
\hline \hline
\end{tabular}

In our case, since the transition changes smoothly from a weakly first-order phase change to a continuous BKT-like transition, the values of $G_{4}$ rapidly depart from unity, but its decrease with sample size is too slow for an extrapolation procedure to be meaningful. In Tables I-III we collect the results for the coexistence properties as determined from the approaches indicated here and in the previous subsection. The results have been crosschecked in the boundary region in which the two approaches can be applied, leading to indistinguishable results within statistical uncertainties.

\section{CONTINUOUS ORDER-DISORDER TRANSITION}

In contrast to the bulk fluid results of Ref. [2], in the confined fluid case, the $N p T$ approach described above no longer leads to estimates for the coexistence densities at sufficiently high temperatures: the transition becomes continuous. One might also interpret this in terms of the presence of an extremely weak first-order approach which cannot be elucidated by the standard $N p T$ simulation analysis. We will see however that a careful analysis of the order parameters, their susceptibilities and heat capacities points to the presence of a BKT-type transition. But first, we will pay attention to the

TABLE II. First-order transition coexistence densities and pressures for the confined MS fluid for interwall separation $h=5 \sigma$. TI stands for thermodynamic integration from $N V T$ simulations.

\begin{tabular}{clllc}
\hline \hline$T^{*}$ & \multicolumn{1}{c}{$\beta p \sigma^{3}$} & \multicolumn{1}{c}{$\rho_{v} \sigma^{3}$} & \multicolumn{1}{c}{$\rho_{l} \sigma^{3}$} & Method \\
\hline 0.80 & $0.062(4)$ & $0.055(3)$ & $0.770(4)$ & TI \\
0.90 & $0.109(20)$ & $0.091(14)$ & $0.703(3)$ & TI \\
1.00 & $0.186(24)$ & $0.144(10)$ & $0.640(7)$ & TI \\
1.10 & $0.274(12)$ & $0.187(11)$ & $0.555(14)$ & TI \\
1.20 & $0.384(4)$ & $0.235(5)$ & $0.509(2)$ & TI \\
1.27 & $0.463(7)$ & $0.274(4)$ & $0.486(2)$ & TI \\
1.35 & $0.551(3)$ & $0.298(6)$ & $0.445(12)$ & TI \\
1.40 & $0.602(16)$ & $0.304(26)$ & $0.453(4)$ & TI \\
1.43 & $0.642(5)$ & $0.315(14)$ & $0.448(6)$ & TI \\
1.45 & $0.658(7)$ & $0.328(10)$ & $0.436(19)$ & TI \\
1.50 & $0.720(6)$ & $0.354(1)$ & $0.439(12)$ & TI \\
1.55 & $0.789(9)$ & $0.366(4)$ & $0.434(14)$ & $N p T$ \\
1.60 & $0.858(6)$ & $0.378(5)$ & $0.438(10)$ & $N p T$ \\
1.65 & $0.921(4)$ & $0.390(4)$ & $0.442(7)$ & $N p T$ \\
\hline \hline
\end{tabular}


TABLE III. First-order transition coexistence densities and pressures for the confined MS fluid for interwall separation $h=8 \sigma$. TI stands for thermodynamic integration from $N V T$ simulations.

\begin{tabular}{clllc}
\hline \hline$T^{*}$ & \multicolumn{1}{c}{$\beta p \sigma^{3}$} & \multicolumn{1}{c}{$\rho_{v} \sigma^{3}$} & \multicolumn{1}{c}{$\rho_{l} \sigma^{3}$} & Method \\
\hline 0.9 & $0.062(34)$ & $0.056(30)$ & $0.761(40)$ & TI \\
1.0 & $0.131(24)$ & $0.107(19)$ & $0.669(40)$ & TI \\
1.1 & $0.197(23)$ & $0.143(10)$ & $0.618(19)$ & TI \\
1.2 & $0.273(10)$ & $0.193(5)$ & $0.551(3)$ & TI \\
1.3 & $0.387(8)$ & $0.230(2)$ & $0.499(14)$ & TI \\
1.4 & $0.477(5)$ & $0.278(24)$ & $0.476(1)$ & TI \\
1.5 & $0.595(10)$ & $0.299(4)$ & $0.454(3)$ & TI \\
1.6 & $0.706(22)$ & $0.334(3)$ & $0.448(24)$ & TI \\
1.7 & $0.809(8)$ & $0.367(6)$ & $0.450(25)$ & TI \\
1.8 & $0.932(6)$ & $0.388(1)$ & $0.446(18)$ & $N p T$ \\
1.9 & $1.072(17)$ & $0.413(3)$ & $0.463(12)$ & $N p T$ \\
2.0 & $1.211(1)$ & $0.440(2)$ & $0.477(1)$ & $N p T$ \\
2.1 & $1.365(3)$ & $0.463(5)$ & $0.495(11)$ & $N p T$ \\
2.2 & $1.540(3)$ & $0.489(3)$ & $0.516(3)$ & $N p T$ \\
\hline \hline
\end{tabular}

determination of the apparent multicritical points, i.e., those points at which the transition switches from first order to a continuous transition. We denote these points as apparent, since the simulation approach cannot discern with sufficient accuracy whether they are "true" multicritical points or critical end points. This remark particularly applies to the quasitwo dimensional case, $h=1.5 \sigma$.

\section{A. Apparent multicritical points}

It is expected that for low values of $h$ ( $h=\sigma$ corresponds to the actual 2D case) the behavior of the system will approach that of the planar system and the I-N transition will transform into a BKT-type continuous transition (at some apparent multicritical temperature $T_{t c}$ ) before reaching the high temperature I-N-S triple point.

The location of the possible multicritical point, $T_{t c}$, of the system requires the use of finite-size scaling techniques, and the simulation of very large systems. One of the more successful approaches to compute critical or multicritical temperatures [24,32-35] relies on the determination of systemsize-dependent pseudocritical points, e.g., $\left\{T_{t c}(L), \mu_{t c}(L)\right\}$ by defining the appropriate order parameters, and then use finite-size scaling procedures to extrapolate the behavior of the infinite system. Details of the methodology used herein can be found in previous works $[1,3]$, so we will just summarize the basic features of the procedure. We carry out computer simulations for different temperatures and volumes using the Grand-Canonical Wang-Landau methodology $[1,24,25]$.

(1) For a given temperature, $T$, and system size $L$ $\equiv(V / h)^{1 / 2}$, we compute the Helmholtz energy function of the system for a large range of densities in the fluid region.

(2) The possible phase transition is located by computing the value of the chemical potential, $\mu_{e}(L, T)$ that maximizes the density fluctuations.

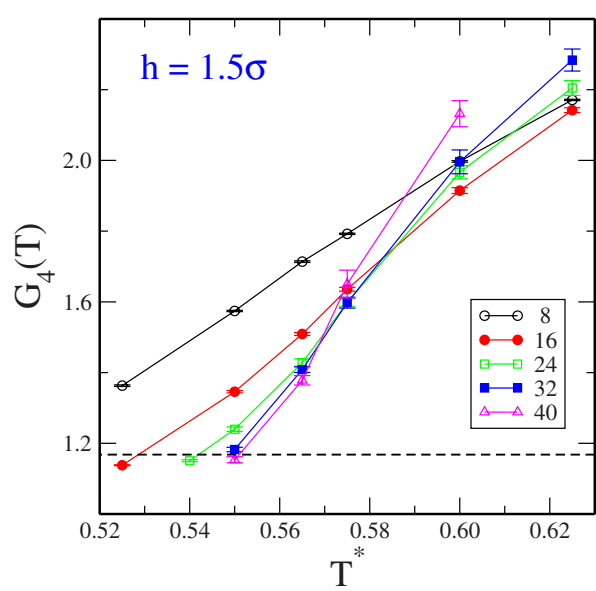

FIG. 3. (Color online) Amplitude ratio $G_{4}(L, T)$ for different system sizes, at constant pore width $h=1.5 \sigma$ from Grand-Canonical Wang-Landau simulations. The dashed horizontal line marks the Universal value for the 2D-Ising model at the same boundary conditions.

(3) Histogram reweighting techniques [29] are used to locate the apparent multicritical temperatures $T_{t c}(L)$, which are defined as the temperatures that fulfill [32]

$$
\frac{\left\langle\left[\delta \rho\left(T, L, \mu_{e}\right)\right]^{4}\right\rangle}{\left\langle\left[\delta \rho\left(T, L, \mu_{e}\right)\right]^{2}\right\rangle^{2}}=G_{4}^{c},
$$

where $\delta \rho \equiv \rho-\langle\rho\rangle$ and $\langle\rho\rangle$ is the average value of the density. For $G_{4}^{c}$ we use the universal value amplitude ratio of the two-dimensional Ising model at the appropriate boundary conditions, i.e., $G_{4}^{c} \simeq 1.168$ [36]. Notice that this particular choice of $G_{4}^{c}$ does not imply that our system does indeed have 2D Ising-like criticality, but is just a practical alternative [31] to define the pseudocritical conditions, taking into account that the proper universality class of our system is not known in advance.

In Fig. 3 we show the $G_{4}$ values for pore width $h / \sigma$ $=1.5$ and various system sizes as a function of the reduced temperature. From this figure it is clear that for the system sizes considered, curves for different values of $L$ do not cross at an unique point.

The system size dependence of the results can be analyzed in terms of scaling laws of the form

$$
T_{t c}(L)=T_{t c}+a_{1}\left(\frac{\sigma}{L}\right)^{1}+a_{2}\left(\frac{\sigma}{L}\right)^{2} .
$$

where the exponent 1 is taken from the analysis of the planar Maier-Saupe model [1,3] and we have added an extra quadratic term which should account for possible deviations stemming from confinement. Similar scaling laws hold for other properties. We found that retaining the linear part of the functional form given in Eq. (13) adequately represents the results (within statistical uncertainty) for pore width $h$ $=1.5 \sigma$ and system sizes $L=16,20,24,28,32,36,40$. The extrapolation leads to the estimate

$$
T_{t c}^{*}=0.565 \pm 0.002 ; \quad \rho_{t c} \sigma^{3}=0.361 \pm 0.002 ;
$$




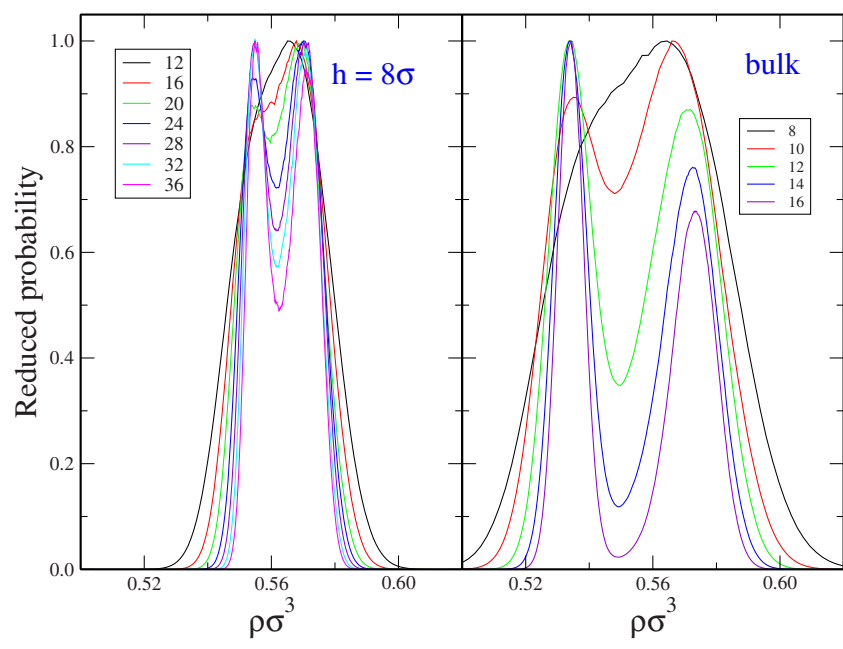

FIG. 4. (Color online) Size dependence of the density probability for the (possible) phase equilibrium conditions for a confined Maier-Saupe fluid with $h=8 \sigma$ (left graph) and for the bulk three dimensional system, both at $T^{*}=2.50$. Curves are labeled according to the system size, $L$, and as $L$ increases the well depth of the bimodal distribution increases (dramatically in the bulk case). The bulk phase is simulated in cubic cells of length $L$ and periodic boundary conditions.

$$
[h=1.5 \sigma ; L \geq 16] .
$$

which is practically the same obtained using the quadratic form and including system sizes starting from $L \geq 8$.

When $h=5 \sigma$, a linear fit (i.e., $a_{2}=0$ ) to Eq. (13) is appropriate for system sizes $L=20,24,28,32,36,40$, leading to

$$
\begin{gathered}
T_{t c}^{*}=1.68 \pm 0.01 ; \quad \rho_{t c} \sigma^{3}=0.417 \pm 0.003 ; \\
{[h=5.0 \sigma, L \geq 20] .}
\end{gathered}
$$

Use of the quadratic correction term in Eq. (13), allows for inclusion of the results for $L=12$, and $L=16$, and yields

$$
\begin{gathered}
T_{t c}^{*}=1.71 \pm 0.02 ; \quad \rho_{t c} \sigma^{3}=0.425 \pm 0.003 ; \\
{[h=5.0 \sigma, L \geq 12] .}
\end{gathered}
$$

For the system with $h=8 \sigma$ we have computed apparent multicritical temperatures for $L=8,10,12,14,16,18,20,22,24$, $26,28,30,32,34,36$, and 40 . The results cannot be fitted to the expression given in Eq. (13) when $a_{2}=0$ for a significant system size range [only considering the results for $L \geq 30$ in Eq. (13) the fitting to $T_{t c}(L)$ is statistically acceptable: $T_{t c}^{(1)}$ $\simeq 2.30 \pm 0.05]$. On the other hand, if the multicritical temperatures are fitted to the quadratic form, one can include system sizes down to $L=20$, and the extrapolated value is $T_{t c}^{(2)}=2.55 \pm 0.10$. Given the large computational cost required to extend the simulations to larger system sizes for $h=8 \sigma$, we have carried out simulations of different system sizes at $T^{*}=2.50$ in order to clarify which of the extrapolations is closer to the actual $T_{t c}$. In Fig. 4 we show the corresponding probability density distributions and compare them with those of the bulk system [2]. It can be seen that the density distributions of the confined system start splitting into two peaks as the system size increases. This would suggest that at $T^{*}=2.50$ there is still a first-order transition, however some caution must be taken, since as stated above, the behavior of the $G_{4}$ parameter for small system sizes does not necessarily depend monotonically on the system size-see Fig. 3-for temperatures slightly above the critical temperature. This is in marked contrast with the situation for the bulk system, in which the well depth of the bimodal distribution increases dramatically with the system size, exhibiting the clear signature of a first-order transition. Given these considerations, we will take for $h=8 \sigma, T_{t c} \simeq 2.50 \pm 0.25$, and $\rho_{t c} \sigma^{3}=0.56 \pm 0.04$.

\section{B. Analysis of the continuous transition}

For temperatures above the $T_{t c}$ calculated above, we enter the region of continuous order-disorder transitions. As usual, the quantity that monitors the order-disorder transition is the largest eigenvalue of Saupe's tensor [37],

$$
Q_{\alpha \beta}(\{j\})=\frac{1}{2 N} \sum_{i=1}^{N}\left(3 s_{i}^{\alpha} s_{i}^{\beta}-\delta_{\alpha \beta}\right) \quad \text { with } \alpha, \beta=x, y, z,
$$

where $\mathbf{s}_{i}$ is the unit vector denoting the orientation of the spin on particle $i$, and the summation is carried out over the $N$ particles of a given system configuration denoted by $\{j\}$. The largest eigenvalue for this tensor is $\lambda_{+}(j)$ (also referred to as the nematic order parameter), and as usual its ensemble average $\lambda_{+}=\Sigma_{j} \lambda_{+}(j) / N_{\text {conf }}$ will be our order parameter, being $N_{\text {conf }}$ the number of configurations considered to evaluate the simulation averages. To perform this analysis we have carried out extensive $N V T$ simulations using 256, 500, 864, 1372, 2048, 2916, and 4000 particles, for a series of temperatures in the neighborhood of the order-disorder transition (first estimated using results from small sample sizes) at given densities above the $\rho_{t c}$ estimated in the previous subsection. Then histogram reweighting [29] is applied in order to determine the values of the order parameter for temperatures other than those directly simulated. In Fig. 5 we observe the sample size dependence of the order parameter for selected densities and three interwall separations. The $h$ $=1.5 \sigma$ case behaves as expected, following the trends already encountered for two-dimensional systems [1,3], i.e., although the slope of the curves increases with system size in the vicinity of the transition temperature, at lower temperatures the order parameter decreases with the system size (this is more clearly seen in the inset) and one might infer that it will vanish in the thermodynamic limit. One can conclude that below the transition temperature the system does not exhibit true long range order (the order parameter would tend to a finite nonzero value if that was the case). As the interwall separation increases, the curves seem to converge, but a rapid look to the insets makes evident that the qualitative behavior is the same for the three interwall separations we have dealt with.

On the other hand, the fact that there is a certain temperature at which the slope of the curves increases rapidly as the sample size is augmented is an indication that some type of 


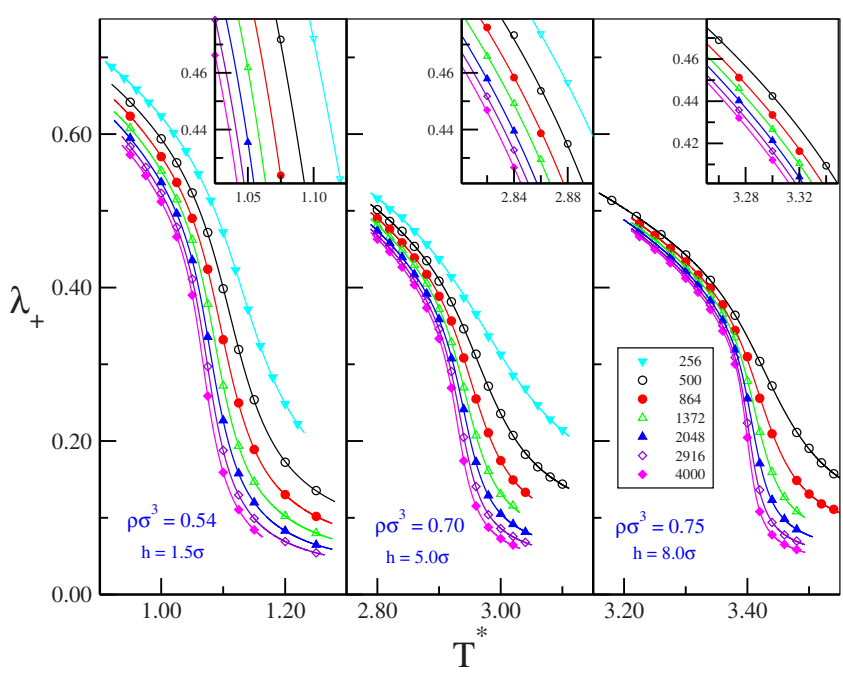

FIG. 5. (Color online) Size dependence of the order parameter in the vicinity of the order-disorder transition of the confined MS fluid for the three interwall separations under consideration. Symbols denote the simulation result and lines are obtained using a histogram reweighting approach.

phase change is taking place. Thus, if one now takes a look at the fluctuation of the order parameter defined by

$$
\chi=N\left(\left\langle\lambda_{+}^{2}\right\rangle-\left\langle\lambda_{+}\right\rangle^{2}\right)
$$

we immediately observe-see Fig. 6-that the curves present a clear maximum whose size dependence indicates that a divergence should occur at the thermodynamic limit. Also, once more we see that for temperatures below that of the maxima, the susceptibility also grows with the sample size. This is more apparent for $h=1.5 \sigma$ and $h=5 \sigma$, but taking a look at the inset of Fig. 6, the curves for $h=8 \sigma$ also indicate that the value of $\chi$ does not converge for temperatures
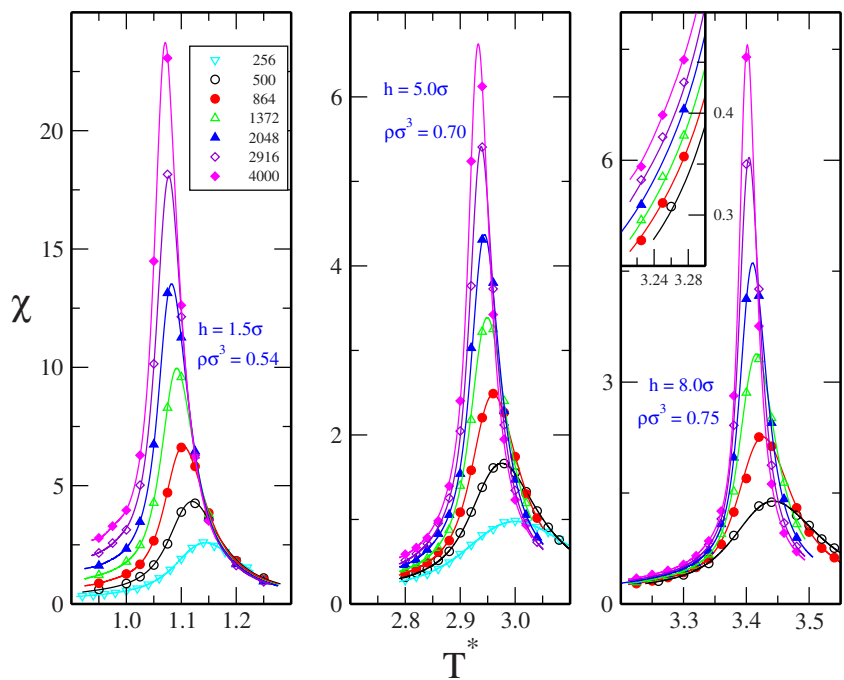

FIG. 6. (Color online) Size dependence of the order-parameter susceptibility in the vicinity of the order-disorder transition of the confined MS fluid for the three interwall separations under consideration. Symbols denote the simulation result and lines are obtained using a histogram reweighting approach.
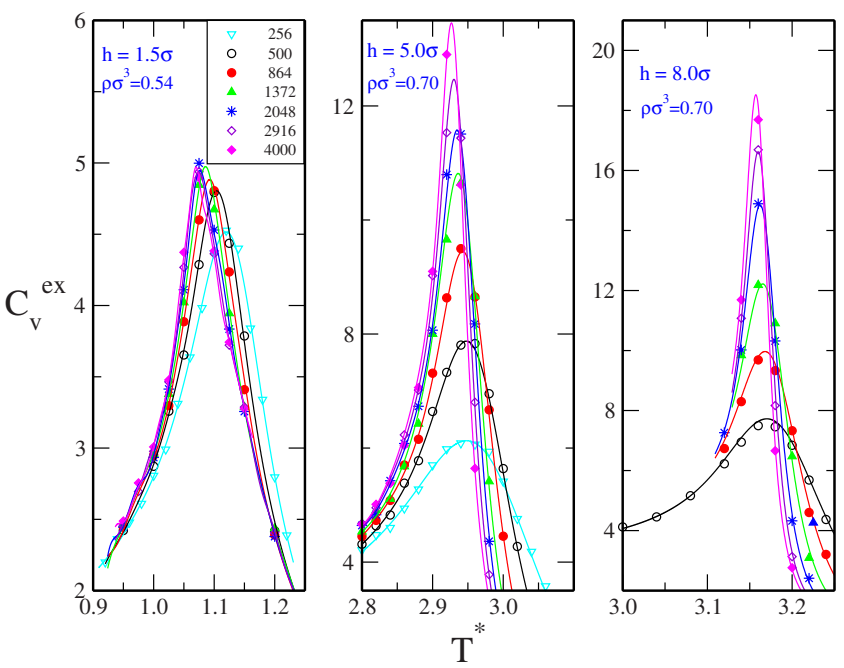

FIG. 7. (Color online) Size dependence of the excess heat capacity in the vicinity of the order-disorder transition of the confined MS fluid for the three interwall separations under consideration. Symbols denote the simulation result and lines are obtained using a histogram reweighting approach.

below that of the transition. As found in Refs. [1,3] we encounter here a situation in which the susceptibility would diverge for any temperature below that of the transition, but without a true long-range order, since the order parameter vanishes in the thermodynamic limit. These features are common with a BKT-type transition. Moreover, the behavior of the excess heat capacity

$$
C_{v}^{e x}=\frac{1}{N T^{* 2}}\left(\left\langle(U / K)^{2}\right\rangle-\langle U / K\rangle^{2}\right)
$$

near the transition temperature-see Fig. 7-points in the same direction. Contrary to the usual behavior at a critical point, we see here that the heat capacity exhibits a maximum, but its size dependence does not indicate a divergence at the thermodynamic limit. This once more fits into the picture of a BKT-type transition [11]. Whether it is strictly a BKT transition or some other type of continuum transition [13] goes beyond the scope of this work, and from a computational standpoint it is nowadays a question out of reach for continuum models. In any case, it is readily seen that the BKT-like character of the transition is more apparent the closer we are to the pure two-dimensional case. According to the behavior observed for growing interwall separations, one should expect that only in the limit of infinite separation the first-order character of the transition is recovered.

In order to give a quantitative estimate for the transition temperatures, following Ref. [38] and our previous works $[1,3]$, we fit the size dependent transition temperatures, $T_{B K T}(L)$ obtained from the maxima of the susceptibility to

$$
T_{B K T}(L)=T_{B K T}+\frac{a}{(c+\ln L)^{2}} .
$$

Additionally, the value of the susceptibility at the maxima is found to scale with sample size as [3] $\chi \propto L^{\gamma / \nu}$, whereby the critical exponent $\gamma / \nu$ can also be determined. Transition tem- 
TABLE IV. Order-disorder transition temperatures and $\gamma / \nu$ exponents for the confined MS fluid.

\begin{tabular}{|c|c|c|c|c|c|c|c|c|}
\hline \multicolumn{3}{|c|}{$h=1.5 \sigma$} & \multicolumn{3}{|c|}{$h=5 \sigma$} & \multicolumn{3}{|c|}{$h=8 \sigma$} \\
\hline$\rho \sigma^{3}$ & $T_{B K T}$ & $\gamma / \nu$ & $\rho \sigma^{3}$ & $T_{B K T}$ & $\gamma / \nu$ & $\rho \sigma^{3}$ & $T_{B K T}$ & $\gamma / \nu$ \\
\hline 0.42 & $0.60(1)$ & $1.68(6)$ & 0.50 & $1.99(1)$ & $1.2(1)$ & 0.55 & $2.43(1)$ & $1.2(2)$ \\
\hline 0.47 & $0.76(1)$ & $1.68(6)$ & 0.55 & $2.23(2)$ & $1.2(1)$ & 0.60 & $2.67(2)$ & $1.4(2)$ \\
\hline 0.50 & $0.87(1)$ & $1.62(4)$ & 0.60 & $2.46(2)$ & $1.3(1)$ & 0.65 & $2.90(3)$ & $1.4(2)$ \\
\hline \multirow[t]{2}{*}{0.54} & $1.00(1)$ & $1.62(1)$ & 0.65 & $2.67(2)$ & $1.3(1)$ & 0.70 & $3.13(2)$ & $1.6(2)$ \\
\hline & & & 0.70 & $2.88(2)$ & $1.4(1)$ & 0.75 & $3.37(3)$ & $1.6(2)$ \\
\hline
\end{tabular}

peratures and critical exponents for a series of densities $\left(\rho>\rho_{t c}\right)$ for each interwall separation are collected in Table IV. The values of $\gamma / \nu$ calculated for $h=1.5 \sigma$ agree well with those of the $\mathrm{R} P^{2}$ model [3] as it should be, and again, do not deviate much from the two-dimensional Ising model value, $\gamma / \nu=7 / 4$. When the interwall separation increases, the value seems to decrease somewhat, particularly at low temperatures when approaching the apparent multicritical points.

In summary, we can say then that for the levels of confinement here considered the systems exhibit a continuous order-disorder transition, in which the ordered phase lacks true long-range order. The dependence of the susceptibilities and heat capacities with the interwall separation indicates that in the transition toward a "regular" first-order phase change no qualitative change as to the nature of the transition or the shape of the phase equilibrium curve occurs. The theoretical analysis of Telo da Gama and Tarazona [20], in fact indicates that in the case of walls that induce no alignment of the spins, the ordered phase will be destroyed by spin waves for any finite interwall separation, $h$. And indeed, as we have seen in our results, no true long-range order is found for any interwall separation we have dealt with and most likely the first-order character of the transition is only recovered for infinite separation limit. On the other hand, Ref. [20] states that in this case there should be no BKT transition. As mentioned before, our results indicate the presence of a continuum order-disorder transition in which the ordered phase lacks true long-range order. Whether this is a true BKT transition or not is a subtle matter beyond the scope of this work.

\section{PHASE DIAGRAM}

Now we can collect the results analyzed in previous sections and construct the phase diagram. The corresponding curves are plotted in Fig. 8, together with the results for the MS spins constrained in a plane (continuous $\mathbb{R} P^{2}$ model), from Ref. [3] and the bulk 3D phase diagram from Ref. [2]. In order to compare the $R P^{2}$ results with those of our quasitwo-dimensional system $(h=1.5 \sigma)$ the volume density has to be transformed into a surface density $\left(\rho_{2 \mathrm{D}}=h \rho_{3 \mathrm{D}}\right)$, which brings the results of the $h=1.5 \sigma$ system to an almost complete agreement with those of the MS fluid confined in a plane. A rapid look at Fig. 8 immediately makes evident that confinement substantially reduces the stability of the condensed (high density) phase. Thus the apparent multicritical temperatures fall as the interwall separation is reduced. In particular when going from $h=5 \sigma$ (4-5 particle layers between the walls, as seen in Fig. 1) to the two-dimensional case, the critical temperature is drastically reduced by a factor 3. Since in our model the walls are inert, this effect of spatial confinement simply results from the fact that the average number of neighbors near the wall is lower than in the bulk, by which the average interaction felt by those particles is also substantially reduced. The tendency to condense must subsequently decrease, and thereby the critical temperatures and the stability of the condensed phase are diminished by

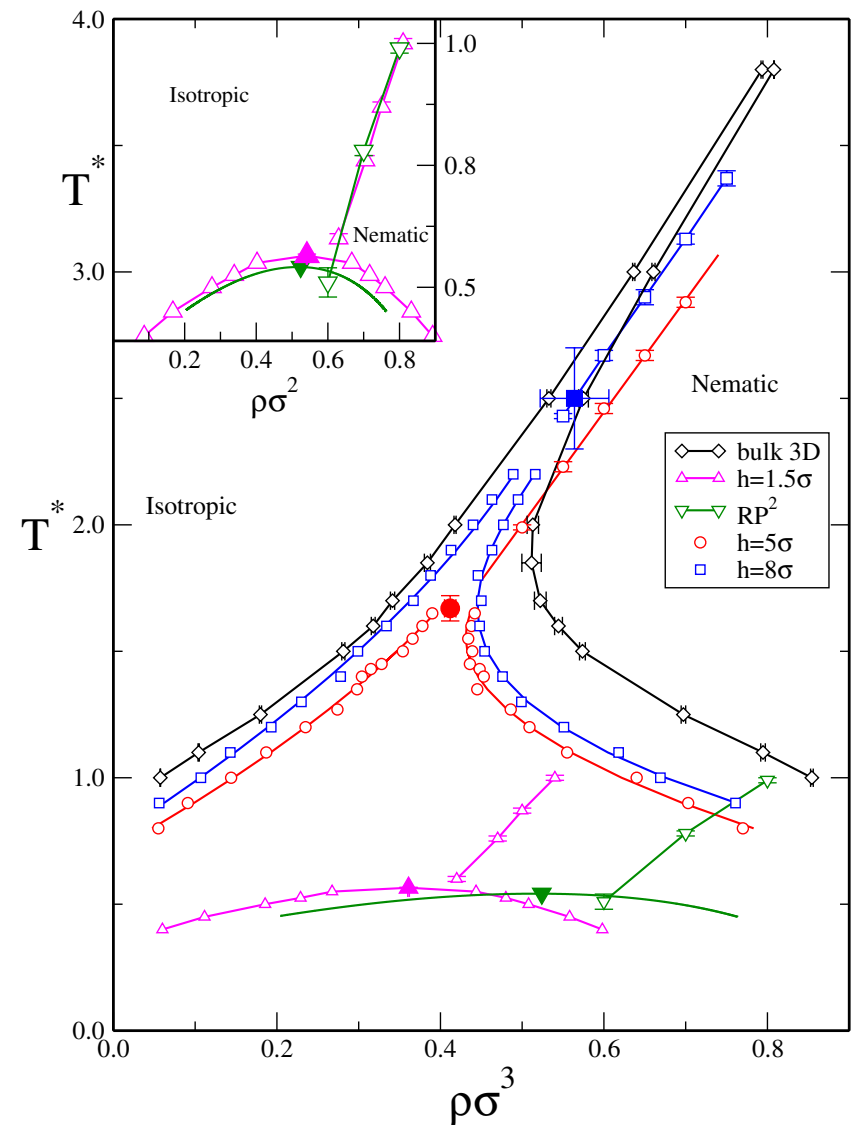

FIG. 8. (Color online) Phase diagram for the MS fluid confined in a plane (continuous $\mathbb{R} P^{2}$ model), confined between two walls separated a distance $h$ (shown in the labels), and 3D bulk phase behavior. Solid symbols indicate the location of the apparent multicritical points. Lines are drawn as guide to the eyes. The inset shows the results for the confined $(h=1.5 \sigma)$ and the $2 \mathrm{D}$ system with the density appropriately rescaled. 
spatial confinement. This effect is well known in fluids confined in porous systems [17]. In the case of the plain vaporliquid equilibrium in fluids inside slit pores of small size, the dependence of the critical temperature on the pore size can be described by classical thermodynamic approaches $[39,40]$. In our case we would expect to obtain a Kelvin-like scaling of the form $T_{c}$ (bulk) $-T_{c}(h) \propto 1 / h$. However, our estimates of the multicritical temperatures do not follow this simple law, and this is easily understood since due to the presence of the order-disorder transition they cannot be directly mapped onto vapor-liquid critical points. Concerning the critical density, we observe that as the fluid is confined, it exhibits a slight decrease, a feature also observed in simple Lennard-Jones fluids confined between purely repulsive walls [41]. Whereas the effect on the critical temperature can be mostly ascribed to spatial confinement, the nature of the wall-fluid interaction is crucial to determine how the critical density is modified upon confinement.

If we focus now on the continuous transition (and the corresponding weakly first-order high temperature isotropicnematic transition of the bulk MS fluid in three dimensions), we observe again that for a given density, the transition temperature decreases as the system is confined, being the change particularly dramatic when reaching the twodimensional limit. Once again, this effect can easily be explained by the decrease in the number of neighbors of particles adjacent to the walls, and the subsequent decrease of the average net interaction felt by their spins. Hence, the tendency to align and form a nematic phase must decrease as the ratio of number of particles close to the walls with respect to those in the bulk increases. Obviously, this result would be different if the walls here not inert with respect to the orientational behavior of the particles, as it would be the case if one deals with a more realistic model for liquid crystals such as the spherocylinder fluid.

In summary, we have presented an extensive study of the influence of spatial confinement on the phase behavior of the MS hard-sphere fluid. Our results show that the change from a first order to a continuous transition when the system is confined all the way down to a two-dimensional plane takes place as the system becomes finite in one of the spatial dimensions, with the two-dimensional character of the transition becoming more apparent as the interwall separation shrinks. For the values of interwall separations considered herein, the continuous transition shares many features in common with the BKT transition, in particular the lack of true long-range orientational order. The effects of confinement on both the BKT transition temperatures and the multicritical temperature can easily be explained as the result of geometric confinement which implies a reduction in the number of neighbors for those layers of fluid adjacent to the walls. An immediate extension of this work should be the incorporation of a spatially varying field acting on the spins, in order to model the influence of the walls on the orientation of the spins. Work on this is planned.

\section{ACKNOWLEDGMENTS}

The authors gratefully acknowledge the support from the Dirección General de Investigación Científica y Técnica under Grant No. MAT2007-65711-C04-04 and from the Dirección General de Universidades e Investigación de la Comunidad de Madrid under Grant No. S0505/ESP/0299 and Program MOSSNOHO-CM.
[1] E. Lomba, C. Martín, N. G. Almarza, and F. Lado, Phys. Rev. E 71, 046132 (2005).

[2] E. Lomba, C. Martín, N. G. Almarza, and F. Lado, Phys. Rev. E 74, 021503 (2006).

[3] E. Lomba, N. G. Almarza, and C. Martín, Phys. Rev. E 76, 061107 (2007).

[4] W. Maier and A. Saupe, Z. Naturforsch. A 14A, 882 (1959).

[5] W. Maier and A. Saupe, Z. Naturforsch. A 15A, 287 (1960).

[6] P. A. Lebwohl and G. Lasher, Phys. Rev. A 6, 426 (1972).

[7] G. R. Luckhurst, S. Romano, and P. Simpson, Chem. Phys. 73, 337 (1982).

[8] G. Luckhurst and P. Simpson, Mol. Phys. 47, 251 (1982).

[9] U. Fabbri and C. Zannoni, Mol. Phys. 58, 763 (1986).

[10] G. R. Luckhurst and S. Romano, Proc. R. Soc. London, Ser. A 373, 111 (1980).

[11] H. Kunz and G. Zumbach, Phys. Rev. B 46, 662 (1992).

[12] A. I. Fariñas-Sanchez, R. Paredes V., and B. Berche, Phys. Lett. A 308, 461 (2003).

[13] R. Paredes V., A. I. Fariñas-Sánchez, and R. Botet, Phys. Rev. E 78, 051706 (2008).

[14] V. Berezinskii, Sov. Phys. JETP 34, 610 (1972).

[15] J. M. Kosterlitz and D. J. Thouless, J. Phys. C 5, L124 (1972).

[16] E. Mondal and S. K. Roy, Phys. Lett. A 312, 397 (2003).
[17] L. D. Gelb, K. Gubbins, R. Radhakrishnan, and M. SliwinskaBartkowiak, Rep. Prog. Phys. 62, 1573 (1999).

[18] D. Cleaver and M. Allen, Mol. Phys. 80, 253 (1993).

[19] M. M. Telo da Gama, P. Tarazona, M. P. Allen, and R. Evans, Mol. Phys. 71, 801 (1990).

[20] M. M. Telo da Gama and P. Tarazona, Phys. Rev. A 41, 1149 (1990).

[21] M. J. P. Nijmeijer and J. J. Weis, Phys. Rev. Lett. 75, 2887 (1995).

[22] U. Wolff, Phys. Rev. Lett. 62, 361 (1989).

[23] M. Allen and D. Tildesley, Computer Simulation of Liquids (Clarendon, Oxford, 1987).

[24] E. Lomba, N. G. Almarza, C. Martin, and C. McBride, J. Chem. Phys. 126, 244510 (2007).

[25] G. Ganzenmüller and P. J. Camp, J. Chem. Phys. 127, 154504 (2007).

[26] E. de Miguel and G. Jackson, J. Chem. Phys. 125, 164109 (2006).

[27] E. de Miguel, N. G. Almarza, and G. Jackson, J. Chem. Phys. 127, 034707 (2007).

[28] W. H. Press, S. A. Teukolsky, W. T. Vetterling, and B. P. Flannery, Numerical Recipes, 3rd ed. (Cambridge University, Cambridge, England, 2007). 
[29] A. M. Ferrenberg and R. H. Swendsen, Phys. Rev. Lett. 63, 1195 (1989).

[30] E. Lomba, C. Martín, and N. Almarza, Mol. Phys. 101, 1667 (2003).

[31] K. Binder, The Monte Carlo Method in Condensed Matter Physics, Topics in Applied Physics Vol. 71, 2nd ed. (SpringerVerlag, Berlin, 1995), pp. 1-22.

[32] D. P. Landau and K. Binder, A Guide to Monte Carlo Simulations in Statistical Physics (Cambridge University Press, Cambridge, England, 2005).

[33] J. Pérez-Pellitero, P. Ungerer, G. Orkoulas, and A. D. Mackie,
J. Chem. Phys. 125, 054515 (2006).

[34] N. G. Almarza, E. Lomba, C. Martin, and A. Gallardo, J. Chem. Phys. 129, 234504 (2008).

[35] N. B. Wilding and P. Nielaba, Phys. Rev. E 53, 926 (1996).

[36] G. Kamieniarz and H. Blöte, J. Phys. A 26, 201 (1993).

[37] A. Saupe, Z. Naturforsch. A 19, 161 (1964).

[38] Y. Tomita and Y. Okabe, Phys. Rev. B 65, 184405 (2002).

[39] A. Vishnyakov, E. M. Piotrovskaya, E. N. Brodskaya, E. V. Votyakov, and K. Yu, Langmuir 17, 4451 (2001).

[40] R. Evans, J. Phys.: Condens. Matter 2, 8989 (1990).

[41] N. G. Almarza (unpublished). 